\title{
TEMPORAL AUTOMATIC EDGE DETECTION OF ECHOCARDIOGRAPHIC IMAGES
}

\author{
L. Torres, A. Gasull \\ Dept. of Signal Theory and Communications \\ E.T.S.I. Telecomunicación - UPC \\ Apdo. 30.002 08080 BARCELONA, SPAIN
}

\begin{abstract}
This paper presents a new fully automatic algorithm that extracts left ventricular epicardial and endocardial borders from 2D echocardiograms.

The algorithm starts with the end systole and end diastole contours obtained through a radial analysis of the image. After some iterative approximations, excellent quality contours for static images are found. At this point, in order to take advantage of the information contained in the temporal domain, the technique is applied to the whole cardiac cycle thus obtaining a complete series of cardiac contours that are used in a further stage to find the left ventricular chamber volume and some other related parameters. The algorithm has been applied to different cardiac views, such a parasternal short axis, apical and mitral, with the same degree of success.
\end{abstract}

\section{INTRODUCTION}

During the last fifteen years echocardiography has experienced widespread acceptance in the evaluation of cardiac disease. This is in large part due to its noninvasive nature and its real time capability for observing both cardiac structure and motion. Two-dimensional echocardiography is capable of providing an integrated overall view of both cardiac structure and motion. Since both global and regional left ventricular function are major variables used to determine prognosis in cardiac disease, there is a great deal of interest in abilities to quantitate function indexes from various types of cardiac imaging techniques. For instance, the analysis of a complete cycle of cardiac images, allows to find left ventricular chamber volume and left ventricular ejection fraction.

Computers have been used mainly to assist in finding cardiac parameters. In this role, they have been adapted in a simple fashion, i.e., manual borders are defined using some type of entry system, and subsequent area and perimeter calculations are made [1], [2]. Some attempts to introduce the computer in the decision-making process have been presented with a moderate amount of success. For instance, Zhang and Geiser [3] have proposed an image processing algorithm for extracting the endocardial border from a series of 2-dimensional echocardiograms (2D echo). Their method is a very efficient method for contour extraction of echographic images but some trained operator has to manually draw the end systole and end diastole contours. Besides, the algorithm fails to detect contours when the cavity is no noise-free. In this context, there is a need for efficient methods of noise removal and posterior parameter extraction.

This work has been supported by NTE, S.A. under European Space Agency contract 7320/87/NL/PP
A main objective of digital processing of echocardiographic images is to improve the signal-to-noise ratio of the video images acquired from the ultrasound equipment. In addition to the white Gaussian noise associated with video signals, there is a large amount of acoustic speckle produced by true signal that arrives at the transducer, but is not associated with return echo from the heart. So, a prime goal is the removal of speckle noise to enhance the image in order to facilitate both visual inspection of the heart and computer parameter extraction. A review of classical methods of left ventricular contour extraction and preprocessing of echocardiographic images is presented in [4].

Once the image has suffered a smoothing process, a contour extraction algorithm may be applied in order to extract cardiac parameters. We have developed a completely fully automatic algorithm for that purpose. The algorithm starts with the end systole and end diastole obtained contours. The main difference with the Zhang and Geiser method is that these first contours are obtained automatically. In order to obtain the two first contours, the image information is changed to polar coordinates and a radial analysis of the image is carried out. Through some iterative approximations, excellent quality contours are obtained. At this point, in order to take advantage of the information contained in the temporal domain, the technique is applied to the whole cardiac cycle thus obtaining a complete series of cardiac contours that are used in a further stage to find the left ventricular chamber volume and some other related parameters.

Temporal processing has been applied due to the fact that ultrasound images contain a lot of correlation thus allowing us to use useful techniques to decrease the search window and speeding up computations. The algorithm has been applied to different cardiac views, such as parasternal short axis, apical and mitral, with the same degree of success.

\section{STATIC ALGORITHM}

As it has been said the starting images of the algorithm are the end systole and end diastole contours. In order to find these first contours we have developed an automatic method which has been already reported [5].The first step is to find the coordinate center in the interior of the cavity where wall contours are being searched. Then the image is converted into polar coordinates by calculating $\mathrm{I}\left(\mathrm{k}_{\mathrm{po}}, 1 \theta_{\mathrm{o}}\right)$ from $\mathrm{I}\left(\mathrm{nx}_{\mathrm{o}}, \mathrm{my}_{\mathrm{o}}\right)$. Bilinear interpolation has been used. Once the image is in polar form, the so called distance function is found, by defining some special characteristic (first maximum, maximum value, etc.) for each radius and drawing the resulting function. A different distance function is evaluated for each contour. Fig. 1 shows the starting function for the inner contour (endocardium) where 
maximum value of each radius has been used to define the distance function. The goal of the algorithm is to find the best possible distance functions for both the inner and outer contours from these first starting distance functions. Fig. 1 also shows the final contour (dashed line).

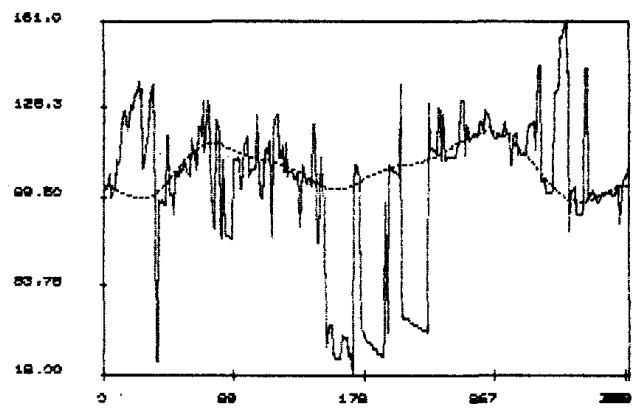

Fig.1. Outer contour distance functions. Continuous line : First approximation. Dashed line : Final result.

Once the first approximation of the distance function is found, the algorithm discriminates which points of this function belong to the contour and which do not. To do this, successive approximations are generated until the contour, or rather its correct distance function is generated. The first step is to determine, from the filtered distance function, a first span of what can be determined to be the actual contour. This first span will lead to follow the contour points. The process of selecting the best span is done through extrapolation of each span along the whole range of the function, and selecting the span that gives the least square error. The extrapolation is performed by a Fourier series approach, taking into account that the function is continuous, periodic and with many continuous derivatives. Through some iterative process all correct spans are incorporated. For the sake of completeness we summarize the static algorithm in Fig. 2

\section{TEMPORAL ALGORITHM}

Once the end systole and end diastole contours have been obtained through the static algorithm, it is logical to apply the method to the whole series of temporal images. If knowledge of the cardiac structure, motion and related parameters is incorporated, it will be possible to find a fast algorithm to find the whole series of temporal contours.

The basic idea is to find a method of contour searching in such a way that starting with an approximate knowledge of the position of the real contour of every one of the images in a temporal ultrasound sequence, it allows to obtain the complete set of real contours.

The fact of knowing the approximate position of the contour, allows to apply the real contour points identification criteria to a restricted area around the previous estimation of the contour position. In this way the interference due to other cardiac structures such as papillary muscles, valves, or even external contour while the internal contour is being obtained (or viceversa), is avoided during the process of detecting the points that belong to the contour wanted to be found. Searching in a reduced region allows that the process of contour detection be faster than in the case of static images where all the points of the image need to be analized.
Input

Preprocessing

Coordinates centre calculation

Image transformation to polar coordinates

Preprocessing

Distance function computation

Median Filtering

Selection of continuous spans

Fourier series approximation of order 0 and 1 for each span

Mean Square error computation between the distance function and each approximation

Selection of the best span by least error approximation

Contour tracking

Point elimination

Fourier series approximation

Interpolation by characteristic searching

Interpolation by a gray-level

searching

Point elimination

Fourier series approximation

\section{Final Contour}

Fig. 2. Automatic contour extraction algorithm for static images

The first step is to preprocess the image in order to eliminate the effects of the noise present in the images during the contour detection. Afterwards, the image is converted into polar coordinates. The best preprocessing results in sequential images have been provided by a $5 * 5$ temporal / spatial median filtering of three consecutive images in polar coordinates. 
In all the images of the sequence, the search of the contour is started based on an estimated position of the real contour. This position is determined from the contours corresponding to the start and end of the sequence - end diastole and end sistoleand assuming that the walls motion is lineal during the cardiac cycle. The criterium of searching the contour points is applied to points around to a specified center determined by the estimated position. This process is applied to every radii of the image that has previously been converted in polar coordinates. The size of the searching region is a parameter that depends on the image in such a way that regions that could lend to wrong points detections - papillary muscles other contours etc. - are avoided. The detected points are then validated and from these points a first approximated contour is built. This first contour will be the starting point for a second searching process. The final contour is obtained from the result of this second search for every one of the images of the sequence.

The contours of end diastole and end sistole are the starting point of the final proposed algorithm for temporal images. These contours are called:

$$
\begin{array}{ll}
R_{d} & i=1, \ldots, N r \\
R_{S} & i=1, \ldots, N r
\end{array}
$$

where $\mathrm{Nr}$ is the number of radiis of the image to be processed; in this case $\mathrm{Nr}=360$. These values represent the distance measured in pixels from the center of coordinates of the image to the point of the contour for each radii $i$

From these values, the estimated contours for the rest of the images (the whole temporal sequence) are obtained by means of a lineal interpolation in the following way:

$$
\begin{aligned}
& R_{n s}^{i}=R_{d}^{i}-\frac{R_{d}^{i}-R_{s}^{i}}{N_{s}-1} \times(n s-1) \\
& R_{n d}^{i}=R_{d}^{i}-\frac{R_{d}^{i}-R_{s}^{i}}{N-N_{s}} \times(N-n d)
\end{aligned}
$$

where $\mathrm{ns}=1, \ldots$, Ns.

nd $=\mathrm{Ns}+\mathrm{1}, \ldots, \mathrm{N}$

$\mathrm{N}=\mathrm{Ns}+\mathrm{Nd}+1$

Ns is the number of images between the instants of end diastole and end sistole, Nd is the number of images between the instants of end sistole and end diastole and $\mathrm{N}$ is the total number of images in the considered sequence. The results of this interpolation give the estimated contours for every one of the images of the sequence.

If the point of the estimated contour is taken as the search center for each radii, the criterium of contour search is applied to a window defined around this defined point. The window size must be introduced as input of the algorithm. The window size must be large enough in order to detect the points that belong to the real contour per each radii, but small enough in such a way that other cardiac structures do not affect the contour detection. For the given images, good results have been obtained using a window size of 10 pixels, i.e., 5 above and 5 below the estimated center.

The applied criteria have been: 1) Detection of the absolute maximum, 2) Maximum of the absolute derivative, 3) Maximum of the positive derivative and 4) First non isolated maximum. The best results in the cavity contour detection have been obtained using the criteria of detection of maximum positive derivative.

In the external contour detection (pericardium) the best results have been obtained using the criteria of the maximum of the absolute value of the derivative.

The criteria of the maximum absolute value in a restricted region provides a good detection in the lower part but since the image contains a lot of noise, it provides a bad detection in the region corresponding to the endocardium, i.e., region between both contours. The detection using the maximum absolute derivative provides good results in the first stage of the searching process. The result of this first search is validated by detecting sets of points through some connectivity criterium, like for instance maximum distance between detected pixels belonging to the same set and minimum length of span (minimum number of detected consecutive points verifying the connectivity criteria in order to consider that set of points valid). The criteria of span detection serves to eliminate points that have been detected in far positions of the real contour. The resulting points of this detection that do not belong to any span are eliminated.

A first approximated contour is built with the validated points. This contour is constructed by finding the Discrete Fourier Transform (DFT) of the validated points, and limiting the resulting spectrum to some specified frequency samples. Good results are obtained with 5 frequency samples. If the inverse DCT is found from these samples, a smoothed contour is obtained that approximates very much the detected points.

Since the detection by derivative means to detect the transitions between very different gray levels, the result of the detected smoothed points always gives a contour that in some regions of the image is smaller than the real contour. This is solved by doing a new search in which the starting point is precisely this first approximated contour instead of the initial estimation obtained by the lineal interpolation from the start and end images of the sequence. The search criterium of this second process is the absolute value. This search is not done around the approximate contour - as in the first search - but starting from the approximate position of the contour per each radii and moving towards the outer position. Good results are obtained with search windows of 4 pixels.

The final contour is obtained building a smoothed contour based on the DFT and inverse DFT with a limited number of frequency samples of the detected points in this second search.

The same method is applied in the detection of the cavity internal contour (endocardium). The decision criteria are the only significative changes. In the first detection the applied criterium is that of detection by the maximum value of the positive derivative. This avoids the strong transitions of gray levels in regions very close to the contour position caused by papillary muscles or valves, in sections corresponding to 
determined regions of the left ventricle in parasternal short axis views.

Once the decision criterium has been applied, the algorithm is exactly the same as that of the external contour.

Fig. 3 shows the final external and internal contours for a temporal sequence of 10 images between end systole and end diastole images.

\section{IV.CONCLUSIONS}

A fully automated algorithm that extracts ventricular endocardial and epicardial borders of temporal parasternal short axis views has been developed. The algorithm may be applied to other echocardiographic views with the same amount of success. Once the external and internal contours are found, ventricular chamber volume and other related parameters can be found.

\section{REFERENCES}

[1] S.Collins, D.J.Skorton "Cardiac Imaging and Image Processing" Mc.Graw-Hill, 1986

[2] D.J. Skorton, et al. "Digital signal and image processing in echocardiography" American Heart journal Vol. 110, $\mathrm{N}^{\mathrm{Q}} 6$ pp.1266-1283, Dec. 1985

[3] L. Zhang, E.A. Geiser "An effective algorithm for extracting serial endocardial borders from two-dimensional echocardiograms" IEEE Trans. Bio. Eng. BME-31, pp. 441-447, 1984

[4] L.Torres et al. "Classical methods of left ventricular contour extraction and preprocessing of echocardiographic images : A review." Ultrasonics International 89, Madrid, 3-7, July 1989

[5] A.Gasull et al. "Automatic left ventricular contour extraction for volume calculation from echocardiographic images" Ultrasonics International 89, Madrid, 3-7 July 1989
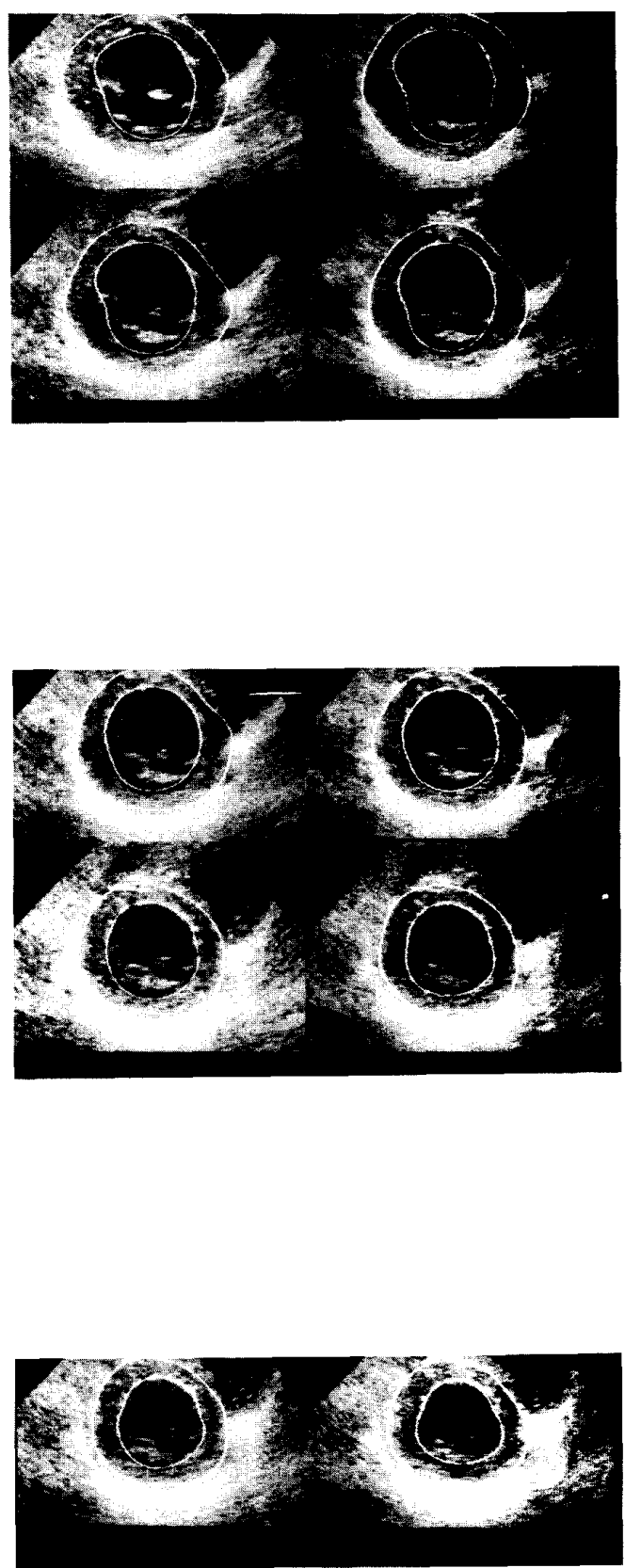

Fig.3. External and Internal contours for an end systole-end diastole cardiac cycle 